\title{
ON THE EIGENFUNCTIONS CORRESPONDING TO THE BANDPASS KERNEL, IN THE CASE OF DEGENERACY*
}

\author{
BY \\ J. A. MORRISON \\ Bell Telephone Laboratories, Murray Hill, New Jersey
}

\begin{abstract}
It has previously been pointed out that the eigenfunctions of the finite integral equation with bandlimited difference kernel satisfy a certain second order linear differential equation, containing one parameter, whose continuous solutions, for discrete values of the parameter, are the prolate spheroidal wave functions. We consider here the finite integral equation with bandpass difference kernel. It is shown that, in the case of degeneracy, one eigenfunction is the continuous solution of a certain fourth order linear differential equation, containing two parameters which must be determined from prescribed conditions. The second eigenfunction is the derivative of the first one.

1. Introduction. In the consideration of bandlimited functions there arises the integral equation

$$
\lambda u(t)=\int_{-1}^{1} \rho(t-s) u(s) d s \equiv K u(t),
$$

where

$$
\rho(t)=t^{-1} \sin c t \equiv \rho_{c}(t) .
$$

It was pointed out by D. Slepian [1] that the self-adjoint differential operator

$$
L(t)=\left\{\frac{d}{d t}\left[\left(1-t^{2}\right) \frac{d}{d t}\right]+\left(\chi-c^{2} t^{2}\right)\right\}
$$

commutes with the integral operator $K$, i.e.,

$$
L(K u)-K(L u) \equiv 0,
$$

when $\rho$ is given by Eq. (1.2). The differential equation $L u=0$ has continuous solutions in the closed $t$ interval $[-1,1]$ only for certain discrete values of the parameter $\chi$, and the corresponding solutions are eigenfunctions of the integral equation (1.1), which may be deduced from Eq. (1.4).

Also of interest is the bandpass kernel

$$
\rho_{a, b}(t)=(b t)^{-1} \sin b t \cos a t ; \quad a>b>0 .
$$

Now Slepian [2] has shown that there is in general no second or fourth order self-adjoint linear differential operator with polynomial coefficients which commutes with the integral operator $K$, when the kernal is given by Eq. (1.5). It is the purpose of this paper to point out that such a fourth order operator does exist if $u$ is even or odd and if, in addition, $u$ is assumed to vanish at the end points, i.e., $u( \pm 1)=0$. The latter condition does not hold in general since it implies that, if $u(t)$ is an eigenfunction of Eq. (1.1) for some $\lambda$, then $u^{\prime}(t)$ is also an eigenfunction for the same value of $\lambda$, as may readily be verified by differentiating Eq. (1.1) and integrating by parts. Thus only in the case of degeneracy

${ }^{*}$ Received June 25, 1962. 
does such an operator exist. But Slepian and Pollak [3] mention that computations indicate the existence of degenerate eigenvalues, for suitable values of $a$ and $b$ in the kernel of Eq. (1.5).

In the next section we illustrate our approach in determining the differential operator by first considering the simpler kernel given by Eq. (1.2).

2. Derivation of the second order operator. We seek a second order self-adjoint differential operator

$$
L[u(t)] \equiv \frac{d}{d t}\left[g(t) \frac{d u}{d t}\right]+f(t) u(t),
$$

which commutes with the integral operator $K[u(t)]$, as defined by Eq. (1.1). Now,

$$
L\{K[u(t)]\}=\int_{-1}^{1} u(s)\left[g(t) \rho^{\prime \prime}(t-s)+g^{\prime}(t) \rho^{\prime}(t-s)+f(t) \rho(t-s)\right] d s .
$$

Also,

$$
K\{L[u(t)]\}=\int_{-1}^{1} \rho(t-s)\left\{\frac{d}{d s}\left[g(s) \frac{d u}{d s}\right]+f(s) u(s)\right\} d s .
$$

Repeated integration by parts gives

$$
\begin{aligned}
K\{L[u(t)]\}=\int_{-1}^{1} u(s)\left[g(s) \rho^{\prime \prime}(t-s)\right. & \left.-g^{\prime}(s) \rho^{\prime}(t-s)+f(s) \rho(t-s)\right] d s \\
+ & {\left[g(s) \frac{d u}{d s} \rho(t-s)+g(s) u(s) \rho^{\prime}(t-s)\right]_{-1}^{1} }
\end{aligned}
$$

From Eqs. (2.2) and (2.4) it follows that $L$ and $K$ commute, i.e., Eq. (1.4) holds, if

$$
g( \pm 1)=0
$$

and

$$
[g(t)-g(s)] \rho^{\prime \prime}(t-s)+\left[g^{\prime}(t)+g^{\prime}(s)\right] \rho^{\prime}(t-s)+[f(t)-f(s)] \rho(t-s)=0 .
$$

We next consider the kernel of Eq. (1.2). It is readily verified that

$$
M_{c}[\rho(t)] \equiv \rho^{\prime \prime}(t)+2 t^{-1} \rho^{\prime}(t)+c^{2} \rho(t)=0, \quad \text { for } \quad \rho=\rho_{c}(t) .
$$

Using Eq. (2.7) to substitute for $\rho^{\prime \prime}(t-s)$ in Eq. (2.6), and equating to zero the resulting coefficients of $\rho(t-s)$ and $\rho^{\prime}(t-s)$, we obtain

$$
[f(t)-f(s)]-c^{2}[g(t)-g(s)]=0,
$$

and

$$
2[g(t)-g(s)]=(t-s)\left[g^{\prime}(t)+g^{\prime}(s)\right] .
$$

Differentiating Eq. (2.9) twice with respect to $t$ leads to $g^{\prime \prime \prime}(t)=0$, and it is readily verified that Eq. (2.9) is indeed satisfied if $g$ is a quadratic function. Invoking Eq. (2.5) and omitting a multiplicative constant,

$$
g(t)=\left(1-t^{2}\right)
$$

Then, from Eq. (2.8), 


$$
f(t)=\left(\chi-c^{2} t^{2}\right),
$$

where $\chi$ is a constant.

We have thus obtained, in a direct manner, the result quoted in the first section. In a second paper [4] we obtain the general solution to Eqs. (2.5) and (2.6), wherein $f, g$, and $\rho$ are assumed to be analytic functions. The bandlimited kernel of Eq. (1.5) is not a member of the limited class of analytic kernels $\rho(t)$ which permit solution. However, from Eqs. (1.2) and (1.5),

$$
\rho_{a, b}(t)=(2 b)^{-1}\left[\rho_{a+b}(t)-\rho_{a-b}(t)\right] .
$$

Also, from Eq. (2.7),

$$
\begin{aligned}
M_{a+b}\left\{M_{a-b}[\rho(t)]\right\}=\rho^{(4)}(t)+ & 4 t^{-1} \rho^{(3)}(t) \\
& +2\left(a^{2}+b^{2}\right)\left[\rho^{\prime \prime}(t)+2 t^{-1} \rho^{\prime}(t)\right]+\left(a^{2}-b^{2}\right)^{2} \rho(t) \\
& =M_{a-b}\left\{M_{a+b}[\rho(t)]\right\} \equiv M_{a, b}[\rho(t)] .
\end{aligned}
$$

Hence, from Eqs. (2.7) and (2.12),

$$
M_{a, b}[\rho(t)]=0, \text { for } \rho=\rho_{a, b}(t) .
$$

It might therefore be expected that there is a fourth order differential operator which commutes with the integral operator, $K$, Eq. (1.1), when $\rho(t)=\rho_{a, b}(t)$. In the next section we investigate this possibility.

3. Derivation of the fourth order operator. We here seek a fourth order self-adjoint differential operator

$$
L[u(t)] \equiv \frac{d^{2}}{d t^{2}}\left[h(t) \frac{d^{2} u}{d t^{2}}\right]+\frac{d}{d t}\left[g(t) \frac{d u}{d t}\right]+f(t) u(t),
$$

which commutes with the integral operator $K[u(t)]$, as defined by Eq. (1.1). Proceeding in a manner similar to that of the previous section, it is found that Eq. (1.4) is satisfied if

$$
\begin{aligned}
{[h(t)-h(s)] \rho^{(4)}(t-s)+} & 2\left[h^{\prime}(t)+h^{\prime}(s)\right] \rho^{(3)}(t-s) \\
& +\left\{\left[h^{\prime \prime}(t)-h^{\prime \prime}(s)\right]+[g(t)-g(s)]\right\} \rho^{\prime \prime}(t-s) \\
& +\left[g^{\prime}(t)+g^{\prime}(s)\right] \rho^{\prime}(t-s)+[f(t)-f(s)] \rho(t-s)=0,
\end{aligned}
$$

and

$$
\begin{aligned}
{\left[\left\{\frac{d}{d s}\left[h(s) \frac{d^{2} u}{d s^{2}}\right]\right.\right.} & \left.+g(s) \frac{d u}{d s}\right\} \rho(t-s)+\left\{h(s) \frac{d^{2} u}{d s^{2}}+g(s) u(s)\right\} \rho^{\prime}(t-s) \\
& \left.+\left\{h(s) \frac{d u}{d s}-\frac{d h}{d s} u(s)\right\} \rho^{\prime \prime}(t-s)+h(s) u(s) \rho^{(3)}(t-s)\right]_{-1}^{1}=0 .
\end{aligned}
$$

We now make use of Eq. (2.14), where $M_{a, b}[\rho(t)]$ is as defined in Eq. (2.13). Substituting for $\rho^{(4)}(t-s)$ into Eq. (3.2) and equating to zero the coefficients of $\rho(t-s)$, $\rho^{\prime}(t-s), \rho^{\prime \prime}(t-s)$ and $\rho^{(3)}(t-s)$, we obtain

$$
\begin{gathered}
{\left[h^{\prime}(t)+h^{\prime}(s)\right](t-s)=2[h(t)-h(s)] ;} \\
\left\{\left[h^{\prime \prime}(t)-h^{\prime \prime}(s)\right]+[g(t)-g(s)]\right\}=2\left(a^{2}+b^{2}\right)[h(t)-h(s)] ;
\end{gathered}
$$




$$
\begin{gathered}
{\left[g^{\prime}(t)+g^{\prime}(s)\right](t-s)=4\left(a^{2}+b^{2}\right)[h(t)-h(s)] ;} \\
{[f(t)-f(s)]=\left(a^{2}-b^{2}\right)^{2}[h(t)-h(s)] .}
\end{gathered}
$$

From Eqs. (3.4) and (3.6),

$$
g(t)=2\left[\left(a^{2}+b^{2}\right) h(t)+\gamma\right],
$$

where $\gamma$ is a constant. It then follows from Eq. (3.5) that $h^{\prime \prime}(t)$ is constant, and hence that $h(t)$ is a quadratic function. This is consistent with Eq. (3.4). From Eq. (3.3) it may be deduced that $h( \pm 1)=0$. Hence, omitting a multiplicative constant,

$$
h(t)=\left(1-t^{2}\right) .
$$

From Eq. (3.7) we then have

$$
f(t)=\left[\delta-\left(a^{2}-b^{2}\right)^{2} t^{2}\right],
$$

where $\delta$ is a constant.

We now return to Eq. (3.3) and satisfy it by the vanishing of the coefficients of $\rho(t-s), \rho^{\prime}(t-s), \rho^{\prime \prime}(t-s)$ and $\rho^{(3)}(t-s)$, at $s= \pm 1$. It is seen that this requires that

$$
u( \pm 1)=0 \text {, }
$$

so that we have degeneracy in the original integral equation, as explained in Section 1. The only other conditions are

$$
h^{\prime}(s) u^{\prime \prime}(s)+g(s) u^{\prime}(s)=0, \text { for } s= \pm 1 .
$$

Now if $u$ is either even or odd, i.e.,

$$
u(t)=u(-t), \text { or } u(t)=-u(-t),
$$

these two conditions reduce to a single condition

$$
u^{\prime \prime}(1)=\gamma u^{\prime}(1),
$$

using Eqs. (3.8) and (3.9). This condition may be regarded as defining $\gamma$.

Thus, to summarize, if

$$
\begin{aligned}
L[u(t)]=\frac{d^{2}}{d t^{2}}\left[\left(1-t^{2}\right) \frac{d^{2} u}{d t^{2}}\right]+\frac{d}{d t}\{ & {\left.\left[\gamma+\left(a^{2}+b^{2}\right)\left(1-t^{2}\right)\right] \frac{d u}{d t}\right\} } \\
& +\left[\delta-\left(a^{2}-b^{2}\right)^{2} t^{2}\right] u,
\end{aligned}
$$

and if $u(t)$ is even or odd and satisfies the boundary conditions of Eqs. (3.11) and (3.14), then $L$ commutes with the integral operator $K$, i.e., Eq. (1.4) holds, where $K[u(t)]$ is defined by Eq. (1.1) with $\rho(t)=\rho_{a, b}(t)$, as defined in Eq. (1.5). We draw our conclusions from this result in the next section.

4. The significance of the commutation of the differential and integral operators.

Suppose now that $L[u(t)]=0$, where $L[u(t)]$ is as defined in Eq. (3.15), and in addition that Eqs. (3.11), (3.13) and (3.14) hold. In order that $u^{(4)}(1)$ should be finite, Eq. (3.15) gives, using Eqs. (3.11) and (3.14),

$$
u^{(3)}(1)=\left[\frac{1}{2} \gamma(\gamma-1)-\left(a^{2}+b^{2}\right)\right] u^{\prime}(1) .
$$

Let $u(t ; \gamma, \delta)$ denote the solution to $L u=0$ which satisfies the boundary conditions of 
Eqs. (3.11), (3.14), and (4.1), with $u^{\prime}(1)=1$, say. It is supposed that $a$ and $b$ are given. Then $u(0 ; \gamma, \delta), u^{\prime}(0 ; \gamma, \delta), u^{\prime \prime}(0 ; \gamma, \delta)$ and $u^{(3)}(0 ; \gamma, \delta)$ are definite functions of $\gamma$ and $\delta$. But, for Eq. (3.13) to hold, we must have

$$
\begin{array}{ll}
u^{\prime}(0 ; \gamma, \delta)=0=u^{(3)}(0 ; \gamma, \delta), & \text { if } u \text { is even; } \\
u(0 ; \gamma, \delta)=0=u^{\prime \prime}(0 ; \gamma, \delta), & \text { if } u \text { is odd. }
\end{array}
$$

Thus we have a dual eigenvalue problem for $\gamma\left(a^{2}, b^{2}\right)$ and $\delta\left(a^{2}, b^{2}\right)$.

Now we have seen that, under the above conditions on $u$, Eq. (1.4) holds. Since $L u=0$, this implies that $L(K u)=0$. Now the equation $L w=0$ has two even, and two odd, linearly independent solutions. At least one of these four solutions cannot remain finite at $t= \pm 1$. But, from Eq. (3.15),

$$
\begin{aligned}
& 0=(w L u-u L w) \\
&=\frac{d}{d t}\left\{w \frac{d}{d t}\left[\left(1-t^{2}\right) \frac{d^{2} u}{d t^{2}}\right]-u\right. \frac{d}{d t}\left[\left(1-t^{2}\right) \frac{d^{2} w}{d t^{2}}\right]+\left(1-t^{2}\right)\left(\frac{d u}{d t} \frac{d^{2} w}{d t^{2}}-\frac{d w}{d t} \frac{d^{2} u}{d t^{2}}\right) \\
&\left.+2\left[\gamma+\left(a^{2}+b^{2}\right)\left(1-t^{2}\right)\right]\left(w \frac{d u}{d t}-u \frac{d w}{d t}\right)\right\} .
\end{aligned}
$$

Hence, if $L v=0$ and $v$ has the same parity as $u$, we obtain on integration

$$
\begin{aligned}
v \frac{d}{d t}\left[\left(1-t^{2}\right) \frac{d^{2} u}{d t^{2}}\right]-u \frac{d}{d t}\left[\left(1-t^{2}\right) \frac{d^{2} v}{d t^{2}}\right]+\left(1-t^{2}\right)\left(\frac{d u}{d t} \frac{d^{2} v}{d t^{2}}-\frac{d v}{d t} \frac{d^{2} u}{d t^{2}}\right) \\
+2\left[\gamma+\left(a^{2}+b^{2}\right)\left(1-t^{2}\right)\right]\left(v \frac{d u}{d t}-u \frac{d v}{d t}\right)=0 .
\end{aligned}
$$

From Eqs. (3.11), (3.14), (4.1), and (4.4) it follows that $v$ is finite at $t=1$.

We have shown that $L(K u)=0$. But, from Eq. (1.5), we see that $K u$ has the same parity as $u$, where

$$
K[u(t)]=\int_{-1}^{1} \rho_{a, b}(t-s) u(s) d s .
$$

Hence,

$$
K[u(t)]=\mu u(t)+v(t),
$$

where $\mu$ and $\nu$ are functions of $a^{2}$ and $b^{2}$ only. Thus we see that only for a specific relationship between $a^{2}$ and $b^{2}$ will we have $\nu=0$, and hence that $u$ is a solution of the integral equation (1.1), with the kernel of Eq. (1.5). Now, since $u^{\prime}(1) \neq 0$, we may take $v^{\prime}(1)=0$ without loss of generality. Since $u(1)=0$, it follows from Eqs. (4.5) and (4.6) that

$$
v(1) \neq 0 \text { and } \int_{-1}^{1} \rho_{a, b}(1-s) u(s) d s=0 \Rightarrow \nu=0 .
$$

It may happen that $v(1)=0$, necessarily. Since, by assumption, $v^{\prime}(1)=0$, it follows that $v^{\prime \prime}(1) \neq 0$ if $v(1)=0$, for this would imply that $v \equiv 0$, since $L v=0$ and $v$ is finite at $t=1$. Hence, from Eqs. (3.14), (4.5), and (4.6),

$$
v(1)=0 \text { and } \int_{-1}^{1}\left[\rho_{a, b}^{\prime \prime}(1-s)-\gamma \rho_{a, b}^{\prime}(1-s)\right] u(s) d s=0 \Rightarrow \nu=0 .
$$


There is thus a straightforward, though lengthy, numerical procedure by which eigenfunctions of the integral equation $K[u(t)]=\lambda u(t)$, where $K[u(t)]$ is defined by Eqs. (1.5) and (4.5), may be obtained in the case of degeneracy, with $u( \pm 1)=0$. Suppose that $b^{2}$ is given. Choosing a value for $a^{2}$, the first step is to find eigenvalues $\gamma\left(a^{2}, b^{2}\right)$ and $\delta\left(a^{2}, b^{2}\right)$, such that $L u=0$, where $L[u(t)]$ is given by Eq. (3.15), and $u$ is subject to Eqs. (3.11), (3.13), (3.14), (4.1), and (4.2). A possible procedure is to integrate $L u=0$ from $t=1$ to $\mathrm{t}=0$ and to minimize (to zero) $\left\{\left[u^{\prime}(0 ; y, \delta)\right]^{2}+\left[u^{(3)}(0 ; \gamma, \delta)\right]^{2}\right\}$, or $\left\{[u(0 ; \gamma, \delta)]^{2}+\left[u^{\prime \prime}(0 ; \gamma, \delta)\right]^{2}\right\}$, according as $u$ is to be even or odd. Having determined $\gamma$ and $\delta$ it is straightforward to compute the other solution $L v=0$, where $v$ has the same parity as $u$. The value of $a^{2}$ must be adjusted, and the above procedure repeated, until the condition of Eq. (4.7), or Eq. (4.8), holds, whichever is appropriate. In the next section we give an analytical discussion of the limiting case $b \rightarrow 0$ in Eq. (1.5). The results obtained could form a basis for the numerical computation for $b>0$, although such computations have not been attempted.

5. The limiting case $b \rightarrow 0$. We here consider the kernel

$$
\rho(t)=\lim _{b \rightarrow 0} \rho_{a, b}(t)=\text { cos at, } \quad a>0,
$$

where $\rho_{a, b}(t)$ is given by Eq. (1.5). The nonzero eigenvalues of the integral equation

$$
\lambda u(t)=\int_{-1}^{1} \cos a(t-s) u(s) d s,
$$

are $\lambda=\left[1+(2 a)^{-1} \sin 2 a\right]$ and $\lambda=\left[1-(2 a)^{-1} \sin 2 a\right]$, with eigenfunctions cos at and sin at, respectively. Thus degeneracy occurs when $a$ is a (positive) integral multiple of $\pi / 2$.

Now, from the results of Sections 3 and 4 , letting $b \rightarrow 0$, we know that if

$$
\begin{gathered}
L u \equiv \frac{d^{2}}{d t^{2}}\left[\left(1-t^{2}\right) \frac{d^{2} u}{d t^{2}}\right]+2 \frac{d}{d t}\left\{\left[\gamma+a^{2}\left(1-t^{2}\right)\right] \frac{d u}{d t}\right\} \\
+\left(\delta-a^{4} t^{2}\right) u=0 ; \quad u(t)= \pm u(-t),
\end{gathered}
$$

and

$$
u(1)=0 ; \quad u^{\prime \prime}(1)=\gamma u^{\prime}(1) ; \quad u^{(3)}(1)=\left[\frac{1}{2} \gamma(\gamma-1)-a^{2}\right] u^{\prime}(1),
$$

then

$$
\int_{-1}^{1} \cos a(t-s) u(s) d s=\mu_{0} u(t)+\nu_{0} v(t)
$$

where $\mu_{0}$ and $\nu_{0}$ are functions of $a^{2}, L v=0$ and $v$ has the same parity as $u$. It is readily verified that the eigenfunctions $\cos$ at, with $a=\left(m+\frac{1}{2}\right) \pi$, and sin at, with $a=(m+1) \pi$, of Eq. (5.2), satisfy Eqs. (5.3) and (5.4) with $\gamma=0$ and $\delta=a^{2}\left(a^{2}-2\right)$. As a point of interest we remark that with the choice, in the notation of Flammer [5], $2 \gamma=\left[\lambda_{\ln }\left(a^{2}\right)-a^{2}\right]$, $\delta=a^{2}\left[\lambda_{1 \mathrm{n}}\left(a^{2}\right)-2\right]$, Eqs. (5.3) and (5.4) are satisfied by $u(t)=\left(1-t^{2}\right)^{1 / 2} S_{1 \mathrm{n}}(a, t)$.

Now $\lambda=0$ is a eigenvalue, of infinite multiplicity, of Eq. (5.2). If we return to the integral equation (1.1), with kernel given by Eq. (1.5), and assume that $u(t) \sim u_{0}(t)+$ $b^{2} u_{1}(t)$ and $\lambda \sim b^{2} \lambda_{1}$, as $b \rightarrow 0$, then we have, in the limit, 


$$
\begin{gathered}
\int_{-1}^{1} \cos a(t-s) u_{0}(s) d s=0 \\
\int_{-1}^{1} \cos a(t-s)\left[u_{1}(s)-\frac{1}{6}(t-s)^{2} u_{0}(s)\right] d s=\lambda_{1} u_{0}(t) .
\end{gathered}
$$

From Eqs. (5.6) and (5.7), the even and odd eigenfunctions, for $\lambda_{1} \neq 0$, have the form (omitting a multiplicative constant)

$$
\begin{aligned}
& u_{0}(t)=p \cos \text { at }+t \sin \text { at, (even) } \\
& u_{0}(t)=q \sin \text { at }+t \cos \text { at, (odd). }
\end{aligned}
$$

From Eq. (5.6)

$$
\begin{aligned}
& p=\left(\frac{\cos 2 a}{2 a}-\frac{\sin 2 a}{4 a^{2}}\right)\left(1+\frac{\sin 2 a}{2 a}\right)^{-1}, \\
& q=\left(\frac{\cos 2 a}{2 a}-\frac{\sin 2 a}{4 a^{2}}\right)\left(1-\frac{\sin 2 a}{2 a}\right)^{-1} .
\end{aligned}
$$

From Eq. (5.7),

$$
\begin{array}{ll}
\lambda_{1}=\frac{1}{3} \int_{-1}^{1} s \sin \text { as } u_{0}(s) d s, & \left(u_{0} \text { e ven }\right) \\
\lambda_{1}=\frac{1}{3} \int_{-1}^{1} s \cos \text { as } u_{0}(s) d s, & \left(u_{0} \text { odd }\right)
\end{array}
$$

Now $u_{0}(1)=0$ when, from Eqs. (5.8) and (5.9),

$$
\begin{array}{ll}
\sin a \cos ^{2} a-a \cos a-2 a^{2} \sin a=0, & \text { ( } \left.u_{0} \text { even }\right) \\
\sin ^{2} a \cos a+a \sin a-2 a^{2} \cos a=0, & \text { ( } \left.u_{0} \text { odd }\right) .
\end{array}
$$

If Eq. (5.11) is satisfied there is degeneracy and $u_{0}^{\prime}(t)$ is also an eigenfunction. It may be verified, using Eqs. (5.8)-(5.10), that the values of $\lambda_{1}$ corresponding to the even and odd eigenfunctions are then equal, and moreover these are the only conditions under which they are equal. The question arises as to whether or not the eigenfunctions, with $u_{0}(1)=0$, in the cases of degeneracy are solutions of the differential eigenvalue problem, Eqs. (5.3) and (5.4). This may readily be verified to be the case. Returning to the integral equation, degeneracy presumably occurs in the eigenvalues of $0\left(b^{2 m}\right)$ as $b \rightarrow 0$, for all positive integers $m$, and presumably the eigenfunctions, with $u_{0}(1)=0$, in all these cases of degeneracy are solutions of the differential eigenvalue problem.

Acknowledgment. I am grateful to H. O. Pollak for bringing this problem to my attention, and for his encouragement.

\section{REFERENCES}

1. D. Slepian, Estimation of signal parameters in the presence of noise, IRE Trans. Infor. Theory, PGIT-3, (1954) 68-89

2. D. Slepian, private communication

3. D. Slepian and H. O. Pollak, Prolate spheroidal wave functions, Fourier analysis and uncertainty-I, BSTJ, 40, (1961) 43-64

4. J. A. Morrison, On the commutation of finite integral operators, with difference kernels, and linear selfadjoint differential operators.

5. C. Flammer, Spheroidal wave functions, Stanford University Press, Stanford, California, 1957. 